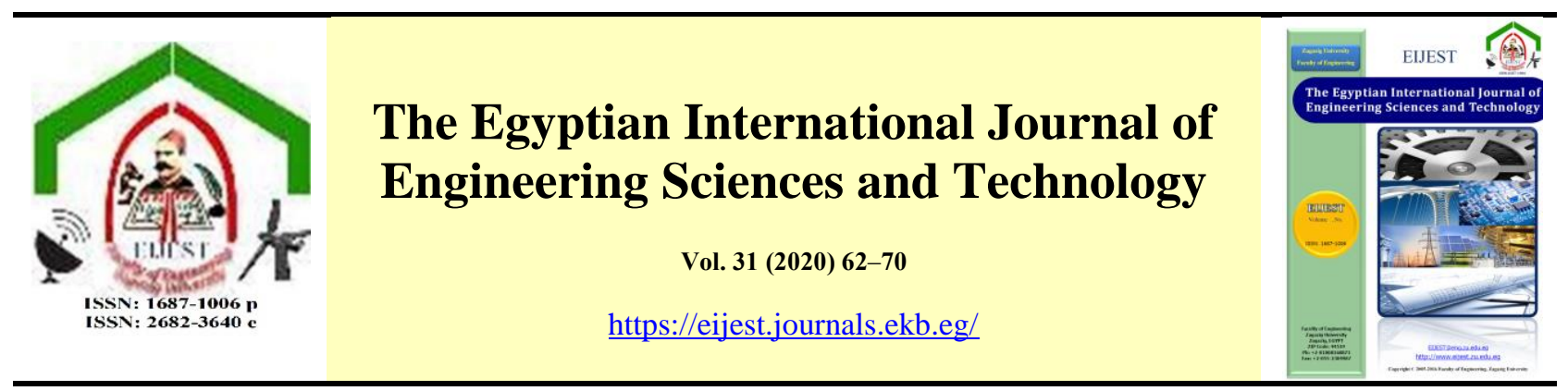

\title{
Computational DIQM Scheme for Solving Nonlinear Volterra Integro- Differential Equations
}

\author{
Norhan A. Mohamed *, Sara I. Abo-Hashem, Salwa A. Mohamed \\ Department of Engineering Mathematics, Faculty of Engineering, Zagazig University, Egypt.
}

\begin{tabular}{ll}
\hline A R T I C L E I N F O & A B S T R A C T \\
\cline { 3 - 3 } $\begin{array}{l}\text { Keywords } \\
\text { Volterra Integro-differential } \\
\text { equation }\end{array}$ & $\begin{array}{l}\text { The authors in this work develop the differential quadrature method (DQM) to } \\
\text { solve the nonlinear Volterra integro-differential equation by introducing an } \\
\text { Differential quadrature } \\
\text { method }\end{array}$ \\
$\begin{array}{l}\text { Integral quadrature method } \\
\text { Exponential convergence } \\
\text { rates }\end{array}$ & $\begin{array}{l}\text { Volterra integro-differential equation into a nonlinear algebraic system of equations } \\
\text { which is solved iteratively by Newton's method. The efficiency of the (DIQM) }\end{array}$ \\
method is examined by solving three examples where the error norms and \\
convergence rates achieve the expected exponential behaviour.
\end{tabular}

\section{Introduction}

In recent years, there has been a clear interest in the integro-differential equations because some of the physical phenomena in engineering and physics fields cannot be described by differential equations only. In many cases, the effects of the past history of the system is needed to be reflected in the model which is described as a function of space and time and these effects of the previous history are ignored. Therefore, the way to overcome this problem is including an integral term in the differential equation to reflect all the preceding states through which the system has passed; that is to say, it is considered as a hereditary phenomenon which leads to integro-differential equations (IDEs) [1, 2]. Integro-differential equations play an important role to describe many processes like physical problems including thermoelastic contact [3], visco-elasticity [4-6], the theory of heat conduction [7-9], fluid dynamics, dropwise condensation and population [10-13]. Analytical solutions of integro-differential equations are hard to compute or they do not exist. Due to this, several numerical methods are developed to get an efficient numerical solution of integro- differential equation by many authors. In [14], an explicit integration method is used for solving a parabolic partial integro-differential equation (PIDE). The numerical solutions by finite element procedures are presented in [15-17]. Volterra equations are classified as IDEs that have been introduced by Vito Volterra (1860-1940). Aggarwal and Gupta [18] applied Kamal Transform for solving Linear Volterra Integro-differential equations of the second kind while in [19], they derived mixed interpolation methods for first and second-order VIDEs with periodic solutions. Also, Brunner et al. in [20] extend recent results for the numerical solution of Volterra integro- differential equations with periodic solution. The Haar wavelets are applied for solving of nonlinear Volterra integral equations and integrodifferential equations in [21] and Legendre wavelet method is developed by Sahu and Ray [22] to approximate the solutions of the system of nonlinear Volterra integro-differential equations. Also, the nonlinear Volterra integro-differential equations with weakly singular kernels are solved by using spectral collocation methods as in [23, 24].

Rohaninasab and his co-workers presented a numerical solution of high-order Volterra integro- 
differential equations by using Legendre collocation method in [25]. Many other numerical methods are used for solving these equations as Aguilar and Brunner in [26] who are using collocation methods to investigate the second-order VIDE in polynomial spline spaces. Chebyshev spectral methods are used to solve the nonlinear Volterra-Hammerstein integral equations [27]. In [28] a finite difference scheme is proposed for the weakly singular kernel of PIDEs. Hyperbolic PIDEs is discussed in [29] by spectral method. The numerical solution of the nonlinear parabolic Volterra PIDE is proposed in [30] by using the finite difference scheme for time and radial basis functions for space discretization. An analysis of Spectral collocation is proposed in [31] for the weakly singular kernel PIDEs. Also, several successive approximation methods for solving integro-differential equations are listed in literature such as Taylor polynomial [32], wavelet-Galerkin method [33], meshless method [34] and Taylor collocation method [35].

One of the most efficient numerical methods which appear in the last decades to solve differential equations is the differential quadrature method (DQM). It was first put by Bellman and Casti in the early 1970s [36], which is used for solving ordinary and partial differential equations by discretizing the derivatives at all the domains. Differential Quadrature Method (DQM) attracted the interest of many authors and because of that, its applications rapidly developed. Many researchers have done different types of DQMs using diverse base functions such as Hermit polynomials [37], spline functions [38], B-spline functions [39], Sinc functions [40].

The advantage of the differential quadrature method appears when it is used to solve boundaryvalue, initial-value, linear or nonlinear differential equations that DQM requires fewer grid points to obtain acceptable accuracy unlike finite difference method (FDM), finite element method (FEM) and finite volume method (FVM) which may need more number of grid points to obtain the solution. The authors in [41-43] succeeded to develop the DQM to solve problems that have an integral term in its differential equation by merging a new integrating matrix operator in the method and obtained differential integral quadrature method (DIQM). Also, by applying DIQM to other applications like nano-beams [44-47], it succeeds to obtain accurate solutions. Therefore, the strategy in this work reposes mainly on establishing an algorithm based on DIQM to obtain the solution of the Volterra integroordinary differential equations.

\section{Problem Formulation}

The authors consider the following nonlinear Volterra integro ordinary differential equation (VIODE):

$\frac{d u}{d t}=f(t)+\mathcal{F}(k), \quad t \in j:=[0, T], \mathrm{u}(0)=\mathrm{a}$.

where; a is constant value and $\mathcal{F}(\mathrm{k})$ is a Volterra integral operator which is defined by:

$$
\mathcal{F}(k)=\int_{0}^{t} \mathcal{K}(t, s) g(u(s)) d s .
$$

The source function $\mathrm{f}$, the nonlinear function $\mathrm{g}$ and kernel function $\mathcal{K}$ are assumed to be sufficiently smooth. This integro-differential equation is called a second kind Volterra integral equation since the unknown function $\mathrm{u}$ appears both inside and outside the integral sign of the equation.

\section{The Solution Procedure}

\section{1. DQM Method}

Let the domain $0 \leq t \leq T$ of a problem be discretized using $N$ nodes; $t_{1}=0, t_{2}, \cdots, t_{N}=T$. Using the DQM, different order derivatives of a function at a given node can be approximated using a weighted sum of the function values at all discrete nodes in that domain. For example, the first derivative of function $f(t)$ at node $t_{i}$ can be approximated using the DQM as follows:

$$
\left.\frac{d f}{d t}\right|_{t=t_{i}} \cong \sum_{j=1}^{N} a_{i j} f_{j}, \quad i=1,2, \cdots, N,
$$

where $f_{j}=f\left(t_{j}\right)$ and $a_{i j}$ denote the corresponding weighting coefficients. The weighting coefficients for the first derivative can be expressed as follows, Shu [48]:

$a_{i j}=\frac{1}{t_{j}-t_{i}}\left(\frac{P_{i}}{P_{j}}\right), \quad i \neq j$ and $a_{i i}=-\sum_{j=1, j \neq i}^{N} a_{i j}^{1}$,

where

$$
P_{i}=\prod_{j=1, j \neq i}^{N}\left(t_{i}-t_{j}\right), i, j=1,2, \cdots, N .
$$

In matrix form, the vector $\boldsymbol{f}=$ $\left[f_{1}, f_{2}, \cdots, f_{n}\right]^{T}$ represents the discrete values $f_{i}=$ $f\left(t_{i}\right)$ at nodes $t_{1}=0, t_{2}, \cdots, t_{N}=T$. According to Eq. (3), the DQM approximation of the first derivative is given by

$$
F=\mathrm{A} f,
$$

where $F=\left[f_{1}, f_{2}, \cdots, f_{n}\right]^{T}, f_{i}^{\prime}=\left(\frac{d f}{d x}\right)_{i} \quad$ and 
$\mathrm{A}=\left[a_{i j}\right]$ is the weighting $(N \times N)$ matrix of the first order derivative that can be easily computed using Eqs. $(4,5)$. The weighting coefficients matrices for higher-order derivatives can be determined via matrix multiplication.

For the purpose of efficiency, the discrete nodes on the domain are distributed according to the Chebyshev- Gauss- Lobatto formula

$$
t_{i}=\frac{T}{2}\left(1-\cos \frac{i-1}{N-1} \pi\right), \quad i=1,2, \cdots, N .
$$

\subsection{The Integral Quadrature Method (IQM)}

Consider a differentiable function $f(t)$ defined on the interval $a \leq t \leq b$. In continuous calculus, if

$$
\frac{d f(t)}{d t}=F(t)
$$

Integrating both sides of Eq.(8), then $\int F(t)=$ $f(t)+c$ and $f(t)$ is called an antiderivative of $F(t)$. In this work, we are interested in the definite integral

$\int_{0}^{T} F(t) d t=(f(T)+c)-(f(0)+c)=f(T)-f(0)$.

It is noticed that its value is independent the arbitrary constant $c$. Returning to DQM, Eq. (6) is discretized in matrix form as $\mathrm{A} \boldsymbol{f}=\boldsymbol{F}$ (see Eq. (6)). To inverse this differentiation process, one can tri writing $\boldsymbol{f}=$ $A^{-1} \boldsymbol{F}$. However matrix $\mathrm{A}$ is singular and hence has no inverse. This is expected and interpreted mathematically since the anti-derivative of a given function is not unique due to the presence integration constant. The main idea behind defining the definite integral matrix operator is to replace the nonexistent $\mathrm{A}^{-1}$ by a pseudo-inverse matrix $\mathrm{A}^{+}$of $\mathrm{A}$. Mohamed [49] has proven that $\mathrm{A}^{+}$acts as anti-derivative operator with respect to the differential operator $A$ such that

$$
\mathrm{A}^{+} \boldsymbol{F}=\boldsymbol{f}+\boldsymbol{C},
$$

where $\boldsymbol{C}$ is a vector with all of its component equals $c=\frac{-1}{N} \sum_{i=1}^{N} f_{i}$. That is $\mathrm{A}^{+}$reverses the derivative operator A but for an additive constant. Note that, in Eq. (10), the $i^{\text {th }}$ component can be written as

$$
\left(\mathrm{A}^{+} \boldsymbol{F}\right)_{i}=\sum_{k=1}^{N} \mathrm{~A}_{i k}^{+} F_{k}=f_{i}+c .
$$

Consequently the definite integral between two nodes $t_{i}, t_{j}$ is approximated as

$$
\int_{t_{i}}^{t_{j}} F(t) d t=f\left(t_{j}\right)-f\left(t_{i}\right) \unrhd \sum_{k=1}^{N}\left(\mathrm{~A}_{j k}^{+}-\mathrm{A}_{i k}^{+}\right) F_{k},
$$

where' $\unrhd$ ' stands for ' is discretized and approximated by'.

Note that the row vector $R_{[i j]}=\left(\mathrm{A}^{+}{ }_{j k}-\mathrm{A}^{+}{ }_{i k}\right), k=$ $1,2, \cdots, N$ is just the difference between the jth and ith rows of matrix $\mathrm{A}^{+}$. Eq. (12) is written in matrix form as

$$
\int_{t_{i}}^{t_{j}} F(t) d t \unrhd \mathrm{R}_{[i j]} \hat{F} .
$$

Next we consider approximation of $\int_{0}^{t} F(t) d t$ , $0<t<T$. Define the integral matrix $R$ as

$$
R=\mathrm{A}^{+}-\overline{\mathrm{A}^{+}},
$$

where $\overline{A^{+}}$is an N-square matrix formed such that each of its rows equals the first row in $\mathrm{A}^{+}$. The definite integral operator $R$ is interpreted through the following expanded form

$$
\left[\begin{array}{c}
\int_{0}^{\mathrm{t}_{1}=0} \mathrm{~F}(\mathrm{t}) \mathrm{dt} \\
\int_{0}^{\mathrm{t}_{2}} \mathrm{~F}(\mathrm{t}) \mathrm{dt} \\
\vdots \\
\vdots \\
\int_{0}^{\mathrm{t}_{\mathrm{N}}} \mathrm{F}(\mathrm{t}) \mathrm{dt}
\end{array}\right] \unrhd\left[\begin{array}{cccccc}
0 & 0 & \cdots & \cdots & \cdots & 0 \\
\mathrm{R}_{21} & \mathrm{R}_{22} & \cdots & \cdots & \cdots & \mathrm{R}_{2 \mathrm{~N}} \\
\vdots & \vdots & & & & \vdots \\
\vdots & \vdots & & & & \vdots \\
\mathrm{R}_{\mathrm{N} 1} & \mathrm{R}_{\mathrm{N} 2} & \cdots & \cdots & \cdots & \mathrm{R}_{\mathrm{NN}}
\end{array}\right]\left[\begin{array}{c}
\mathrm{F}_{1} \\
\mathrm{~F}_{2} \\
\vdots \\
\mathrm{F}_{\mathrm{N}}
\end{array}\right],
$$

where element $R_{i j}=\mathrm{A}^{+}{ }_{i j}-\mathrm{A}^{+}{ }_{1 j}$. The definite integral operator $\mathcal{R}$ has the following properties

$$
\begin{gathered}
\int_{0}^{t} F(t) d t \unrhd \mathcal{R} \boldsymbol{F}, \\
\int_{0}^{t_{i}} F(t) d t \unrhd \overline{\mathcal{R}}_{i} \boldsymbol{F} .
\end{gathered}
$$

where $\bar{R}_{i}$ is the ith row of $R$.

\subsection{DIQM for Integro-Differential Equations}

Consider the nonlinear Volterra integro ordinary differential equation Eqs.(1,2) with the independent variable $0<t<T$. Using the Chebyshev-GaussLobatto distribution Eq. (7), the domain is discretized by $N$-points. Let the discrete vectors $\boldsymbol{t}, \boldsymbol{u}, \boldsymbol{f}$ for $t$, unknown function $u(t)$, and known function $f(t)$, be respectively

$$
t=\left[\begin{array}{c}
t_{1} \\
t_{2} \\
\vdots \\
t_{N}
\end{array}\right], u=\left[\begin{array}{c}
u_{1} \\
u_{2} \\
\vdots \\
u_{N}
\end{array}\right], f=\left[\begin{array}{c}
f_{1} \\
f_{2} \\
\vdots \\
f_{N}
\end{array}\right]
$$

Let the $N \times N$ matrices $A$ and $R$ be respectively the first order differential operator and the definite integral operator. First, consider the integral term 


$$
\mathbb{I}(t)=\int_{0}^{t} \mathcal{K}(t, s) \mathrm{g}(u(s)) d s
$$

Note that the i-th component of $\mathbb{I}(t)$ is $\mathbb{I}_{i}=$ $\int_{0}^{t_{i}} \mathcal{K}\left(t_{i}, s\right) g(u(s)) d s$, its DIQM discretization is

$I_{i} \unrhd \bar{R}_{i}\left[\begin{array}{c}K\left(t_{i}, t_{1}\right) g\left(u_{1}\right) \\ K\left(t_{i}, t_{2}\right) g\left(u_{2}\right) \\ \vdots \\ K\left(t_{i}, t_{N}\right) g\left(u_{N}\right)\end{array}\right]=\sum_{1=1}^{N} R_{i l} K\left(t_{i}, t_{1}\right) g\left(u_{1}\right)$.

Accordingly, $\mathbb{I}(t)$ can be discretized by DIQM as

$\mathbb{I}(t)=\int_{0}^{t} \mathcal{K}(t, \tau) \mathrm{g}(u(\tau)) d \tau \unrhd\left[\begin{array}{c}\sum_{j=1}^{N} R_{1 j} \mathcal{K}\left(t_{1}, t_{j}\right) \mathrm{g}\left(u\left(t_{j}\right)\right. \\ \vdots \\ \sum_{j=1}^{N} R_{i j} \mathcal{K}\left(t_{i}, t_{j}\right) \mathrm{g}\left(u\left(t_{j}\right)\right. \\ \vdots \\ \sum_{j=1}^{N} R_{K j} \mathcal{K}\left(t_{K}, t_{j}\right) \mathrm{g}\left(u\left(t_{j}\right)\right.\end{array}\right]=$

$\left(\mathcal{R} \circ \overline{\mathcal{K}}\left(\mathbb{T}, \mathbb{T}^{T}\right)\right) \boldsymbol{g}(\boldsymbol{u})$.

It must be mentioned that if $\mathbb{I}(t)$ is a part in an integro-differential equation then its discrete algebraic system is nonlinear. To solve the resulting nonlinear system by Newton method one has to compute the Jacobian matrix for the system. To compute the Jacobian matrix IJ corresponding to the integral term II defined in Eq.(19), we first compute its general element $\mathbb{J}_{i j}$ by differentiating the right hand size of Eq.(20) to get

$$
\mathbb{I}_{i j}=\frac{\partial \mathbb{\Pi}_{i}}{\partial u_{j}}=R_{i j} \mathcal{K}\left(t_{i}, t_{j}\right) g^{\prime}\left(u_{j}\right) .
$$

In matrix form, the Jacobian matrix for the right hand side of Eq. (20) is obtained as where

$$
\mathbb{J}=R \circ \overline{\mathcal{K}}\left(\mathbb{T}, \mathbb{T}^{T}\right) \circ g^{\prime}(U),
$$

$$
\begin{gathered}
U=\left[\begin{array}{cccc}
u_{1} & u_{2} & \cdots & u_{K} \\
u_{1} & u_{2} & \cdots & u_{K} \\
\vdots & \vdots & & \vdots \\
u_{1} & u_{2} & \cdots & u_{K}
\end{array}\right], \quad g^{\prime}=\frac{d g}{d u^{\prime}} \\
\mathbb{T}=\left[\begin{array}{cccc}
t_{1} & t_{1} & \cdots & t_{1} \\
t_{2} & t_{2} & \cdots & t_{2} \\
\vdots & \vdots & & \vdots \\
t_{K} & t_{K} & \cdots & t_{K}
\end{array}\right] .
\end{gathered}
$$

Hadamard matrix operator ' $o$ ' is the element by element operator defined for matrices $\mathcal{A}, \mathcal{B}, \mathcal{C}$ having the same dimensions such that $\mathcal{C}=\mathcal{A} \circ \mathcal{B}$ implies that $\mathcal{C}_{\mathrm{ij}}=\mathcal{A}_{\mathrm{ij}} \mathcal{B}_{\mathrm{ij}}$. In Eq. (20), $\overline{\mathcal{K}}\left(\mathbb{T}, \mathbb{T}^{T}\right)$ is a square $N$-matrix with general element $\overline{\mathcal{K}}_{i j}=\mathcal{K}\left(\mathbb{T}_{i j}, \mathbb{T}_{i j}^{T}\right)$.

This approach enables DIQM to transform integrodifferential equation Eq. (1) into a system of nonlinear algebraic equations.

$$
\mathcal{F}=A \boldsymbol{u}-\boldsymbol{f}-B g(\boldsymbol{u})=0 .
$$

With the Jacobian matrix

$$
\mathcal{T}=A-B \circ g^{\prime}(U),
$$

where matrix $B=R \circ \overline{\mathcal{K}}\left(\mathbb{T}, \mathbb{T}^{T}\right)$.

\section{Numerical Results}

In this section, we prove the effectiveness of our approach which is based on DIQM to obtain the approximate solution of nonlinear Volterra IODEs. All of the numerical computations are performed using MATLAB R2018b and the accuracy is measured for the proposed method by using the following two error norms definitions:

$$
\begin{aligned}
L_{2} & =\sqrt{\sum_{i=1}^{N}\left(\mathrm{u}_{\text {computed }}(i)-\mathrm{u}_{\text {exact }}(i)\right)^{2}}, \\
L_{\infty} & =\max _{i}\left|\mathrm{u}_{\text {computed }}(i)-\mathrm{u}_{\text {exact }}(i)\right| .
\end{aligned}
$$

where, $N$ is the number of discretized points. Also, the authors provide the convergence rate for all numerical examples where the numerical error is computed by the exponential order $O\left(C^{N}\right)$ for $0<C<1$. The convergence rate is faster when the value of $C$ is smaller and this is achieved if the solution is smooth [50]. The convergence parameter $C$ can be evaluated by:

$$
C=e^{\frac{\ln \left(E r r_{1} / E r r_{2}\right)}{\left(N_{1}-N_{2}\right)}},
$$

where, $\operatorname{Err}_{1}$ and $\operatorname{Err}_{2}$ are the error norms computed at the domain which is discretized by $N_{1}$ and $N_{2}$ of grid points, respectively.

\section{Example 1}

We consider the following nonlinear VIODE [51]:

$\frac{d u}{d t}=f(t)+\int_{0}^{t} t s e^{-u^{2}(s)} d s, \quad t \in[0,1]$

where $f(t)=1-\frac{t}{2}+\frac{t}{2} e^{-t^{2}}$ with initial condition $u(0)=0$ and exact solution $u(t)=t$. 
Applying the proposed DIQM, Eq. (29) is discretized into a nonlinear algebraic system in the form of Eq.

(25) where

$\boldsymbol{g}(u)=\left[e^{-u_{1}^{2}}, e^{-u_{2}^{2}}, \cdots, e^{-u_{K}^{2}}\right]^{T}, B=R \circ \overline{\mathcal{K}}\left(\mathbb{T}, \mathbb{T}^{T}\right)$.

In this example, $\mathcal{K}(t, s)=t s$, then

$$
\overline{\mathcal{K}}\left(\mathbb{T}, \mathbb{T}^{T}\right)=\mathbb{T} \circ \mathbb{T}^{T}=\left[\begin{array}{cccc}
t_{1} t_{1} & t_{1} t_{2} & \cdots & t_{1} t_{K} \\
t_{2} t_{1} & t_{2} t_{2} & \cdots & t_{2} t_{K} \\
\vdots & \vdots & & \vdots \\
t_{K} t_{1} & t_{K} t_{2} & \cdots & t_{K} t_{K}
\end{array}\right] .
$$

The Jacobian for this algebraic system is given by Eq. (26). Note that since $g^{\prime}(u)=-2 u e^{-u^{2}}$, then

$$
g^{\prime}(U)=-2\left[\begin{array}{cccc}
u_{1} e^{-u_{1}^{2}} & u_{2} e^{-u_{2}^{2}} & \cdots & u_{K} e^{-u_{K}^{2}} \\
u_{1} e^{-u_{1}^{2}} & u_{2} e^{-u_{2}^{2}} & \cdots & u_{K} e^{-u_{K}^{2}} \\
\vdots & \vdots & & \vdots \\
u_{1} e^{-u_{1}^{2}} & u_{2} e^{-u_{2}^{2}} & \cdots & u_{K} e^{-u_{K}^{2}}
\end{array}\right]
$$

Table 1: $L_{2}$ and $L_{\infty}$ error norms and the convergence rates for Example 1 at $t=1$.

\begin{tabular}{cccc||cc}
\hline $\boldsymbol{N}$ & $\boldsymbol{M}$ & $L_{2}$ & $\mathbf{C}$ & $L_{\infty}$ & $\mathbf{C}$ \\
\hline $\mathbf{4}$ & 5 & $4.49 \mathrm{E}-04$ & - & $3.67 \mathrm{E}-04$ & - \\
$\mathbf{6}$ & 5 & $3.46 \mathrm{E}-06$ & 0.2963 & $2.62 \mathrm{E}-06$ & 0.2907 \\
$\mathbf{8}$ & 5 & $8.39 \mathrm{E}-08$ & 0.3947 & $5.16 \mathrm{E}-08$ & 0.3746 \\
$\mathbf{1 0}$ & 4 & $1.20 \mathrm{E}-09$ & 0.3459 & $7.02 \mathrm{E}-10$ & 0.3415 \\
$\mathbf{1 2}$ & 4 & $1.34 \mathrm{E}-11$ & 0.3251 & $7.25 \mathrm{E}-12$ & 0.3188 \\
$\mathbf{1 4}$ & 4 & $1.22 \mathrm{E}-13$ & 0.3090 & $6.11 \mathrm{E}-14$ & 0.3029 \\
$\mathbf{1 6}$ & 5 & $8.71 \mathrm{E}-16$ & 0.2905 & $4.44 \mathrm{E}-16$ & 0.2920 \\
\hline
\end{tabular}

The convergence results are presented in Table 1 . The $L_{2}$ and $L_{\infty}$ error norms are reported for different numbers of grid points $\mathrm{N}$. The number $M$ of iteration steps in Newton method required to reduce the error norms below $\epsilon=10^{-15}$ is also reported. To demonstrate the spectral convergence rate, the convergence parameter $\mathrm{C}$ is computed according to Eq. (28) and presented in Table 1 showing that $0.29<C<0.4<1$. The results illustrates that accurate results are obtained using few grid points and that $\mathrm{N}=16$ is sufficient to reduce the error below the computer round off error.

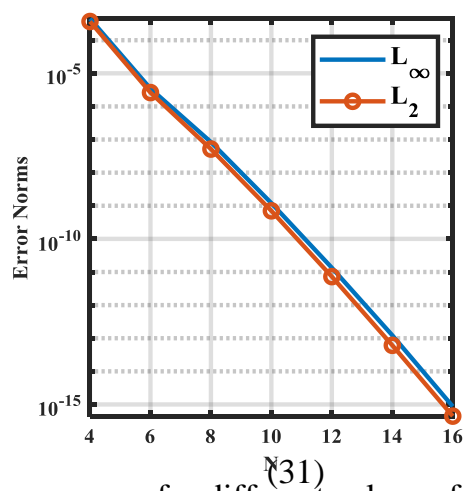

Fig. 1: Error norms for different values of $\mathrm{N}$ at $\mathrm{t}=1$ of Example 1.

These results are also presented in Fig. 1 on a semi$\log$ scale where the error logarithm is plotted versus the number of grid points. For both of the $L_{2}$ and $L_{\infty}$ error norms, the nearly straight line plot indicates that the errors decay exponentially. The $L_{2}$ error norms computed at different times in the interval $0.2<t<$ 1 are plotted in Fig. 2 for N 324 ) 8, 16. It is observed that although the error increases with time, the solution is still accurate in this range of time.

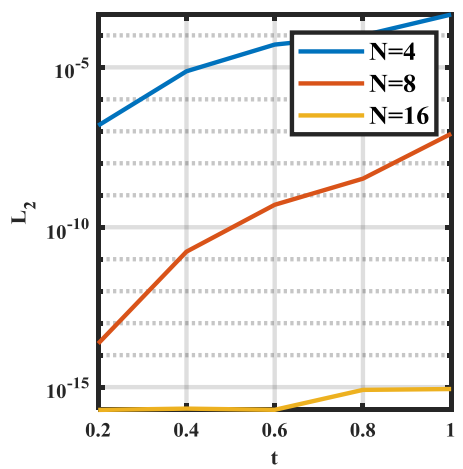

Fig. 2: $L_{2}$ for different values of time and $\mathrm{N}$ for Example 1.

\section{Example 2}

Consider the following nonlinear VIODE [51]:

$\frac{d u}{d t}=f(t)+\int_{0}^{t}(t-s) \ln (1+u(s)) d s, \quad t \in[0,1]$

where,

$$
\begin{aligned}
& f(t)=1 / 24\left(8+9 t^{2}+\frac{12}{\sqrt{1+t}}-8 \sqrt{1+t}-\right. \\
& 4 t\left(-6+5 \sqrt{1+t}-12 t^{2} \ln (1+\sqrt{1+t})\right)
\end{aligned}
$$

with the initial condition $\mathrm{u}(0)=1$, and the exact solution is given by $u(t)=\sqrt{1+t}$. 
By following the same steps in example (1), we can get the Jacobian matrix of this example by Eq. (26) where:

$$
g^{\prime}(U)=\left[\begin{array}{cccc}
\frac{1}{1+u_{1}} & \frac{1}{1+u_{2}} & \cdots & \frac{1}{1+u_{K}} \\
\frac{1}{1+u_{1}} & \frac{1}{1+u_{2}} & \cdots & \frac{1}{1+u_{K}} \\
\vdots & \vdots & & \vdots \\
\frac{1}{1+u_{1}} & \frac{1}{1+u_{2}} & \cdots & \frac{1}{1+u_{K}}
\end{array}\right] .
$$

In Table $2, L_{2}$ and $L_{\infty}$ error norms are Listed for different numbers of grid points $\mathrm{N}$. Also, the convergence rate results are presented in Table 2 where its range is $0.34<C<0.4<1$. This example needs $M=10$, number of iteration steps in Newton method, to reduce the error norms to $\epsilon=10^{-13}$. We need only grid points $\mathrm{N}=16$ to obtain accurate results.

Table 2: $L_{2}$ and $L_{\infty}$ error norms and the convergence rate for Example 2 at $t=1$.

\begin{tabular}{cccc||cc}
\hline $\boldsymbol{N}$ & $\boldsymbol{M}$ & $L_{2}$ & $\mathbf{C}$ & $L_{\infty}$ & $\mathbf{C}$ \\
\hline $\mathbf{4}$ & 10 & $1.29 \mathrm{E}-03$ & - & $8.09 \mathrm{E}-04$ & - \\
$\mathbf{6}$ & 10 & $1.80 \mathrm{E}-05$ & 0.3441 & $1.17 \mathrm{E}-05$ & 0.3472 \\
$\mathbf{8}$ & 10 & $3.62 \mathrm{E}-07$ & 0.3763 & $2.15 \mathrm{E}-07$ & 0.3677 \\
$\mathbf{1 0}$ & 10 & $8.13 \mathrm{E}-09$ & 0.3872 & $4.39 \mathrm{E}-09$ & 0.3782 \\
$\mathbf{1 2}$ & 10 & $1.94 \mathrm{E}-10$ & 0.3930 & $9.64 \mathrm{E}-11$ & 0.3849 \\
$\mathbf{1 4}$ & 10 & $4.81 \mathrm{E}-12$ & 0.3968 & $2.22 \mathrm{E}-12$ & 0.3895 \\
$\mathbf{1 6}$ & 10 & $1.23 \mathrm{E}-13$ & 0.4002 & $5.31 \mathrm{E}-14$ & 0.3932 \\
\hline
\end{tabular}

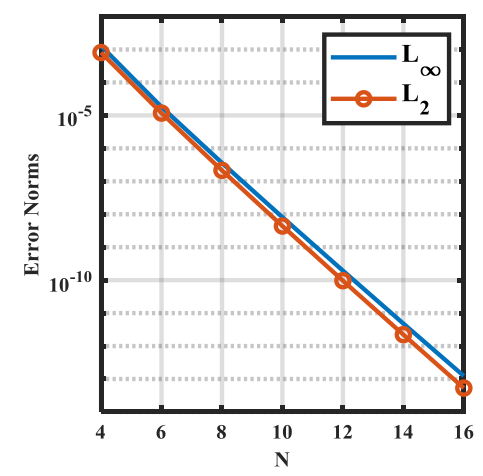

Fig. 3: Error norms for different values of $\mathrm{N}$ at $\mathrm{t}=1$ of Example 2.

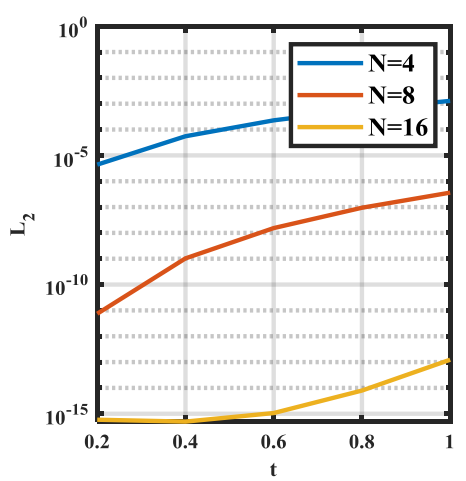

Fig. 4: $L_{2}$ for different values of time and $\mathrm{N}$ for Example 2.

The $L_{2}$ and $L_{\infty}$ error norms are plotted in Fig. 3, the error logarithm on a semi-log scale versus the number of grid points, and the results indicate that the errors decay exponentially. In Fig. 4, the $L_{2}$ error norms are plotted for $\mathrm{N}=4,8,16$ which computed in the interval $0.2<t<1$. As in example 1 , the accuracy is still achieved even with an increase of errors.

\section{Example 3}

We investigate the following nonlinear VIODE [52]:

$$
\frac{d u}{d t}=f(t)+\int_{0}^{t} e^{t-s} u^{3}(s) d s, \quad t \in[0,1]
$$

where, $f(t)=\frac{3}{2} e^{t}-\frac{1}{2} e^{3 t}$ with initial condition $u(0)=1$, and the exact solution is given by $u(t)=$ $e^{t}$.

Also, the Jacobian matrix in this example is obtained as we can get it in the previous examples. And $g^{\prime}(u)=3 u^{2}$, then:

$$
g^{\prime}(U)=3\left[\begin{array}{cccc}
u_{1}^{2} & u_{2}^{2} & \cdots & u_{K}^{2} \\
u_{1}^{2} & u_{2}^{2} & \cdots & u_{K}^{2} \\
\vdots & \vdots & & \vdots \\
u_{1}^{2} & u_{2}^{2} & \cdots & u_{K}^{2}
\end{array}\right]
$$

Table $3: L_{2}$ and $L_{\infty}$ error norms and the convergence rate for Example 3 at $\mathrm{t}=1$.

\begin{tabular}{cccc||cc}
\hline$N$ & $M$ & $L_{2}$ & $\mathrm{C}$ & $L_{\infty}$ & $\mathrm{C}$ \\
\hline $\mathbf{4}$ & 10 & $1.85 \mathrm{E}-01$ & - & $1.72 \mathrm{E}-01$ & - \\
$\mathbf{6}$ & 9 & $2.71 \mathrm{E}-04$ & 0.1957 & $2.23 \mathrm{E}-04$ & 0.1897 \\
$\mathbf{8}$ & 7 & $8.85 \mathrm{E}-07$ & 0.2390 & $6.49 \mathrm{E}-07$ & 0.2321 \\
$\mathbf{1 0}$ & 9 & $2.12 \mathrm{E}-09$ & 0.2213 & $1.41 \mathrm{E}-09$ & 0.2161 \\
\hline
\end{tabular}




\begin{tabular}{cccc||cc}
\hline $\mathbf{1 2}$ & 10 & $3.59 \mathrm{E}-12$ & 0.2027 & $2.20 \mathrm{E}-12$ & 0.1986 \\
$\mathbf{1 4}$ & 10 & $6.92 \mathrm{E}-15$ & 0.2096 & $4.00 \mathrm{E}-15$ & 0.2064 \\
& & & & & \\
$\mathbf{1 6}$ & 8 & $5.89 \mathrm{E}-15$ & 0.9604 & $3.11 \mathrm{E}-15$ & 0.9391 \\
\hline
\end{tabular}

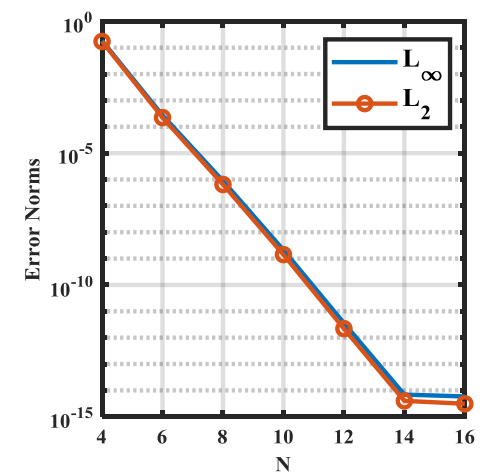

Fig. 5: Error norms for different values of $\mathrm{N}$ at $\mathrm{t}=1$ of Example 3.

As in the previous examples, Table 3 presented that the convergence rate results range from $0.19<C<$ $0.96<1$ of this example. The $L_{2}$ and $L_{\infty}$ error norms need the numbers of grid points $\mathrm{N}=16$ to obtain the error norms of $\epsilon=10^{-15}$. Figs. 5 shows that the $L_{2}$ and $L_{\infty}$ error norms are still decay exponentially and the accuracy is still achieved although the error increases with time as shown in Fig. 6.

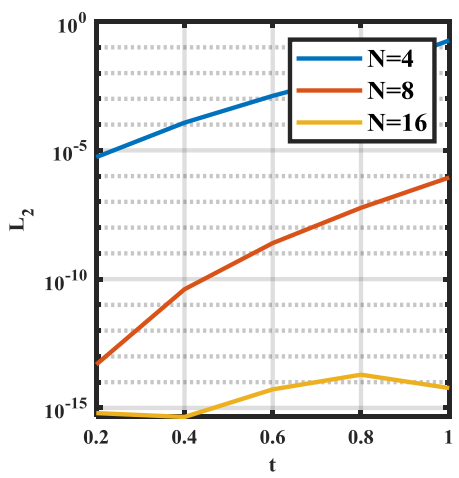

Fig. 6: $L_{2}$ for different values of time and $\mathrm{N}$ for Example 3.

\section{Conclusion}

In this paper, the authors presented the differential integral quadrature method (DIQM) by combining an integration matrix operator with the differentiation matrix in (DQM) method. The obtained method (DIQM) is applied for solving the nonlinear Volterra integro-differential equation (VIDE). The results of the three provided examples illustrate that the proposed method is efficient and achieve the expected exponential behavior of accuracy by using a few numbers of grid points. In future work, the proposed method (DIQM) is easy to implement for solving other kinds of integrodifferential equations.

\section{References}

[1] G. Yanik, G. Fairweather, "Finite element methods for parabolic and hyperbolic partial integro-differential equations", Nonlinear Analysis: Theory, Methods \& Applications, Vol. 12, pp. 785-809, 1988.

[2] F. Fakhar-Izadi, M. Dehghan, "Fully spectral collocation method for nonlinear parabolic partial integrodifferential equations", Applied Numerical Mathematics, Vol. 123, pp. 99-120, 2018.

[3] W. Allegretto, J.R. Cannon, Y. Lin, "A parabolic integro-differential equation arising from thermoelastic contact", Discrete \& Continuous Dynamical Systems-A, Vol. 3 (2), pp. 217-234, 1997.

[4] C.M. Dafermos, "An abstract Volterra equation with application to linear viscoelasticity", Journal of Differential Equations, Vol. 7, pp. 554-569, 1970.

[5] A.S. Lodge, M. Renardy, J.A. Nohel, "Viscoelasticity and Rheology", Academic Press, New York, 1985.

[6] M. Renardy, W. Hrusa, J. Nohel, "Mathematical Problems in Viscoelasticity", Longman, Boston, 1987.

[7] M. Gurtin, A. Pipkin, "A general theory of heat conduction with finite wave speeds", Archive for Rational Mechanics and Analysis, Vol. 31, pp. 113-126, 1968.

[8] S.O. Londen, J.A. Nohel, "A nonlinear Volterra integrodifferential equation occurring in heat flow", The Journal of Integral Equations, Vol. 6, pp. 11-50, 1984.

[9] R. MacCamy, "An integro-differential equation with application in heat flow", Quarterly of Applied Mathematics, Vol. 35, pp. 1-19, 1977.

[10] I. Abdul, "Introduction to Integral Equations with Application", Wiley, NewYork, NY, USA, 1999.

[11] S. Shaw and J. R. Whiteman, "Adaptive space-time finite element solution for Volterra equations arising in viscoelasticity problems", Journal of Computational and Applied Mathematics, Vol. 125, pp. 337-345, 2000.

[12] V. Volterra, "Theory of Functionals And of Integral And Integro- Differential Equations", Dover Publications, Inc., New York, NY, USA, 1959.

[13] A. J. Jerri, "Introduction to Integral Equations with Applications", Marcel Dekker, New Yourk, NY, USA, 1999.

[14] A.S. Vasudeva Murthy, J.G. Verwer, "Solving parabolic integro-differential equations by an explicit integration method", Journal of computational and applied mathematics, Vol. 39, pp. 121-132, 1992.

[15] Y. Lin, V. Thomeé, L. B. Wahlbin, "Ritz-Volterra projections to finite element spaces and applications to integro-differential and related equations", SIAM Journal on Numerical Analysis, Vol. 28, pp. 1047-1070, 1991.

[16] A. K. Pani, V. Thomeé, L. B. Wahlbin, "Numerical methods for hyperbolic and parabolic integro differential 
equations", Journal of Integral Equations and Applications, Vol. 4, pp. 533-584, 1992

[17] C. Chen, P. G. Shih Tsimin, "The Finite Element Methods for Integro-Differential Equations", World Scientific, London, 1997

[18] S. Aggarwal, A.R. Gupta, "Solution of linear Volterra integro-differential equations of second kind using Kamal transform", Journal of Emerging Technologies and Innovative Research, Vol. 6(1), pp. 741-747, 2019.

[19] H. Brunner, A. Makroglou, R. Miller, "Mixed interpolation collocation methods for first and second order Volterra integro-differential equations with periodic solution", Applied numerical mathematics, Vol. 23(4), pp. 381-402, 1997.

[20] H. Brunner, A. Makroglou, R. Miller, "On mixed collocation methods for Volterra integral equations with periodic solution", Applied Numerical Mathematics, Vol. 24(2-3), pp. 115-130, 1997.

[21] Ü. Lepik, "Haar wavelet method for nonlinear integrodifferential equations", Applied mathematics and Computation, Vol. 176(1), pp. 324-333, 2006.

[22] P.K. Sahu, S.S. Ray, "Legendre wavelets operational method for the numerical solutions of nonlinear Volterra integro-differential equations system", Applied mathematics and computation, Vol. 256, pp. 715-723, 2015.

[23] Z. Gu, "Spectral collocation method for weakly singular Volterra integro-differential equations", Applied Numerical Mathematics, Vol. 143, pp. 263-275, 2019.

[24] Y. Yang, Y. Chen, "Spectral collocation methods for nonlinear Volterra integro-differential equations with weakly singular kernels", Bulletin of the Malaysian Mathematical Sciences Society, Vol. 42(1), pp. 297-314, 2019.

[25] N. Rohaninasab, K. Maleknejad, R. Ezzati, "Numerical solution of high-order Volterra-Fredholm integrodifferential equations by using Legendre collocation method", Applied Mathematics and Computation, Vol. 328, pp. 171-188, 2018.

[26] M. Aguilar, H. Brunner, "Collocation methods for second-order Volterra integro-differential equations", Applied numerical mathematics, Vol. 4, pp. 455-470, 1988.

[27] G.N. Elnagar, M. Kazemi, "Chebyshev spectral solution of nonlinear Volterra-Hammerstein integral equations", Journal of computational and applied mathematics, Vol. 76, pp. 147-158, 1996.

[28] T. Tang, "A finite difference scheme for partial integrodifferential equations with a weakly singular kernel", Applied numerical mathematics, Vol. 11, pp. 309-319, 1993.

[29] S.K. Chung, M.G. Park, "Spectral analysis for hyperbolic integro-differential equations with a weakly singular kernel", Journal of the Korean Society for Industrial and Applied Mathematics, Vol. 2, pp. 31-40, 1998.

[30] Z. Avazzadeh, Z.B. Rizi, F.M. Maalek Ghaini, G.B. Loghmani, "A numerical solution of nonlinear parabolic-type Volterra partial integro-differential equations using radial basis functions", Engineering analysis with boundary elements, Vol. 36, pp. 881-893, 2012.

[31] C.H. Kim, U.J. Choi, "Spectral collocation methods for a partial integro-differential equation with a weakly singular kernel", The ANZIAM Journal, Vol. 39, pp. 408-430, 1998.

[32] K. Maleknejad, Y. Mahmoudi, "Taylor polynomial solution of high-order nonlinear Volterra-Fredholm integro-differential equations", Applied Mathematics and Computation, Vol. 145, pp. 641-653, 2003.

[33] K. Maleknejad, F. Mirzaee, "Using rationalized Haar wavelet for solving linear integral equations", Applied Mathematics and Computation, Vol. 160( 2), pp. 579587, 2005.

[34] M. Dehghan, R. Salehi, "The numerical solution of the non-linear integro-differential equations based on themeshless method", Journal of Computational and Applied Mathematics, Vol. 236(9), pp. 2367-2377, 2012.

[35] A. Karamete, M. Sezer, "A Taylor collocation method for the solution of linear integro-differential equations", International Journal of Computer Mathematics, Vol. 79( 9), pp. 987-1000, 2002.

[36] R. Bellman, J. Casti, "Differential quadrature and longterm integration", Journal of mathematical analysis and Applications, Vol. 34(2), pp. 235-238, 1971.

[37] A. Krowiak, "Hermite type radial basis function-based differential quadrature method for higher order equations", Applied Mathematical Modelling, Vol. 40(3), pp. 2421-2430, 2016.

[38] A. Başhan, "A mixed algorithm for numerical computation of soliton solutions of the coupled $\mathrm{KdV}$ equation: Finite difference method and differential quadrature method", Applied Mathematics and Computation, Vol. 360, pp. 42-57, 2019.

[39] A. Başhan et al., "A new perspective for quintic Bspline based Crank-Nicolson-differential quadrature method algorithm for numerical solutions of the nonlinear Schrödinger equation", The European Physical Journal Plus, Vol. 133(1), pp. 12, 2018.

[40] A. Korkmaz, İ. Dağ, "Shock wave simulations using sinc differential quadrature method", Engineering Computations, 2011.

[41] N. Mohamed, M.A. Eltaher, S.A. Mohamed, L.F. Seddek, Numerical analysis of nonlinear free and forced vibrations of buckled curved beams resting on nonlinear elastic foundations, International Journal of Non-Linear Mechanics, Vol. 101, pp. 157-173, 2018.

[42] N. Mohamed, M.A. Eltaher, S.A. Mohamed, L.F. Seddek, "Periodic and Nonperiodic Modes on Postbuckling and Nonlinear Vibration of Beams Attached with Nonlinear Foundations", Applied Mathematical Modelling, Vol. 75, pp. 414-445, 2019.

[43] M. A. Attia, S. A. Mohamed, "Coupling effect of surface energy and dispersion forces on nonlinear sizedependent pull-in instability of functionally graded micro-/nanoswitches", Acta Mechanica, Vol. 230, pp. 1181-1216, 2019.

[44] M. A. Attia, R. A. Shanab, S. A. Mohamed, N. A. Mohamed, "Surface energy effects on the nonlinear free vibration of functionally graded Timoshenko nanobeams based on modified couple stress theory", International Journal of Structural Stability and Dynamics, Vol. 19(11), 2019

[45] M. A. Attia, R. A. Shanab, S. A. Mohamed, N. A. Mohamed, "Effect of Microstructure and Surface Energy on the Static and Dynamic Characteristics of FG Timoshenko Nanobeam Embedded in an Elastic 
Medium", Journal of Nano Research, Vol. 61, pp 97117, 2020.

[46] R. A. Shanab, S. A. Mohamed, N. A. Mohamed, M. A. Attia, "Compressive investigation of vibration of sigmoid and power law FG nanobeams based on surface elasticity and modified couple stress theories", Acta Mechanica, pp. 1-34, 2020.

[47] M. Eltaher, S. A. Mohamed, "Buckling and stability analysis of sandwich beams subject to varing axial loads", steel and composite structures, Vol. 34(2), pp. 241-260, 2020.

[48] C. Shu, "Differential Quadrature and Its Application in Engineering", Springer-Verlag, London, 2000.

[49] S. A. Mohamed, "A fractional differential quadrature method for fractional differential equations and fractional eigenvalue problems", Math Meth Appl Sci, 2020. In press. https://doi.org/10.1002/mma.6753

[50] L. N. Trefethen, "Spectral Method in MATLAB", SIAM, Philadelphia, PA.

[51] M. H. Daliri Birjandi, J. Saberi-Nadjafi, A. Ghorbani, "An Efficient Numerical Method for a Class of Nonlinear Volterra Integro-Differential Equations", Journal of Applied Mathematics, 2018.

[52] K. Kim, "A novel semi-analytical approach for solving nonlinear Volterra integro-differential equations", Applied Mathematics and Computation, Vol. 263, pp. 25-35, 2015. 